\title{
Waterborne polyurethane-polyacrylate dispersions of mixed type
}

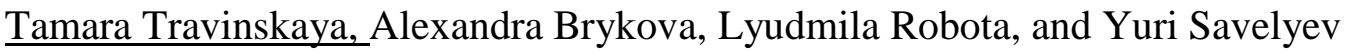

Department of heterochain polymers and interpenetrating networks, Institute of macromolecular chemistry, NAS of Ukraine, UKRAINE, Kiev, Kharkovskoe sh,48, E-mail: travinskaya-tamara@rambler.ru

\begin{abstract}
A series of waterborne, aggregatively stable PUI/PA dispersions has been obtained by combining in the mixture both type of polymers. The effects of component ratio on various properties of PUI/PA, such as particle size, surface tension, rheological behavior, physic-mechanical properties and thermodynamical compatibility have been investigated.
\end{abstract}

Keywords - waterborne polyurethane, polyacrylate, dispersion, mixture, compatibility, composition.

\section{Introduction}

In recent years, the production of dispersions, emulsions and latexes has been widely developed through the development and implementation of resource-saving and environmentally friendly technologies for their production. However, the growing demands on the complex of properties force to create new compositions. One of the way to solve this problem is to change the properties of polymers by modifying them with other polymers, including by mixing them. Among many dispersions and latexes polyurethane and polyacrylate in terms of their production and use occupy one of the leading places. Polyurethane ionomers (PUI) are extremely promising. The presence in their macrochain of ionic centers and water as a liquid phase, solves the problem of environmental protection. Polyacrylate aqueous dispersions (PA) are also widely used as film formers in various sectors of the economy. Obtaining dispersions of the mixed type has the advantage that opens the possibility of "combining" in the binary film of thermodynamically incompatible polymers and improving the characteristics of existing materials. The paper studies the properties of PUI/PA dispersions and based films, depending on the components' ratio.

\section{Materials}

PUI was obtained on the basis of POTMG (MM-1000), TDI (mixture of 2,4-2,6 isomers) in a ratio of 65:35. Isophthalic acid dihydrazide was used as chain extender, pyromelitic acid dianhydride - as the carrier of ionic groups. As the acrylate component was used an aqueous dispersion of a copolymer of ethylester of acrylic acid, nitrile of acrylic acid and methacrylic acid in the ratio 84:13:3, respectively. PUI/PA dispersions of the mixed type were obtained by introducing of from 1 to $50 \mathrm{wt} \%$ PA into PUI latex.

\section{Results}

The properties of PUI, PA dispersions, PUI/PA mixtures and based films are shown in table 1.

Table 1 Properties of PUI, PA, PUI/PA and based films

\begin{tabular}{|c|c|c|c|c|c|r|c|}
\hline \multicolumn{2}{|c|}{$\begin{array}{c}\text { Compositio } \\
\mathrm{n}, \mathrm{wt} \%\end{array}$} & \multicolumn{3}{|c|}{ Properties of dispersions } & \multicolumn{3}{c|}{ Properties of films } \\
\hline $\begin{array}{c}\text { PU } \\
\text { I }\end{array}$ & PA & $\mathrm{pH}$ & $\begin{array}{c}\mathrm{r}_{\mathrm{av}} \\
\mu \mathrm{km}\end{array}$ & $\begin{array}{c}\delta \\
\mathrm{mN} / \mathrm{m}\end{array}$ & $\begin{array}{c}\text { Water } \\
\mathrm{abs}, \%\end{array}$ & $\begin{array}{c}\sigma, \\
\mathrm{MPa}\end{array}$ & $\begin{array}{c}\varepsilon, \\
\%\end{array}$ \\
\hline 100 & 0 & 7,38 & 0,56 & 37,34 & 18,7 & 23 & 500 \\
\hline 95 & 5 & 7,06 & 0,43 & 36,32 & 19,3 & 25 & 600 \\
\hline 90 & 10 & 6,86 & 0,38 & 38,35 & 28,0 & 30 & 680 \\
\hline 80 & 20 & 6,65 & 0,41 & 36,32 & 20,1 & 28 & 630 \\
\hline 70 & 30 & 6,35 & 0,58 & 36,32 & 27,7 & 18 & 550 \\
\hline 50 & 50 & 6,02 & 0,64 & 36,83 & 24,7 & 15 & 500 \\
\hline 0 & 100 & 5,36 & 0,06 & 32,00 & 76,0 & 18 & 450 \\
\hline
\end{tabular}


The choice of the optimal amount of PA affects not only the stability of dispersions, but also the strength characteristics of the films and the value of their water absorption. It is advisable to obtain PUI/PA with a PA content of not more than $20 \%$, since an increase in the proportion of the acrylate component leads to a loss of strength. At $10 \%$ content of the acrylate component, the value of surface tension $(\delta)$ is greater than the individual components. The higher the surface tension of the mixture, the stronger the intermolecular interaction of the components. The increased tensile strength of the binary film of this composition is in agree with the obtained data. The average particle size $\left(r_{a v}\right)$ of the mixtures almost does not change with the change of composition, which confirms the absence of aggregation processes. According to rheological studies, all studied mixtures belong to structured systems and are the non-Newton liquids with thixotropic properties. The effective viscosity exceeds the additive values up to almost $20 \%$ of the PA content. Within the same compositions, the tensile strength and relative elongation exceed the parameters of the original components. This may be due to the compatibility of the components in mixtures of these compositions. The calculated values of the free mixing energy showed that for mixtures in which the PA content does not exceed $20 \%$ are negative, which indicates the thermodynamic compatibility of the components within this concentration range. When the PA content increases by more than $20 \%$, the free mixing energy changes the sign to the opposite (positive), which corresponds to the two-phase state of the system. The reason for the compatibility in the studied polymer mixture is probably the formation of hydrogen bonds between the urethane group of PUI and the ester group of PA. However, compatibility also depends on the competition between the enthalpy and entropy of mixing. The micrographs of the original latexes and their mixtures $90 / 10$ presented in Fig. 1 indicate a more homogeneous structure of the binary PUI/PA (90/10) film. The average value of the particle size in this composition $\mathrm{r}_{\mathrm{av}}(90 / 10)$ is $0,38 \mu \mathrm{km}$, which is less than in other PUI/PA ratios.

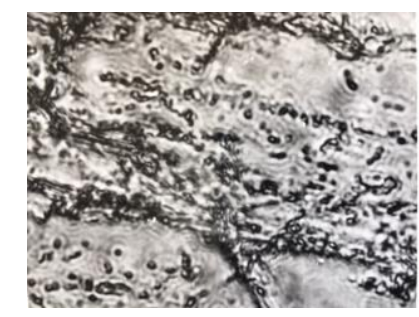

PUI

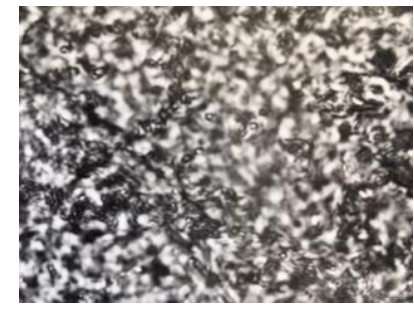

PUI/PA 90/10

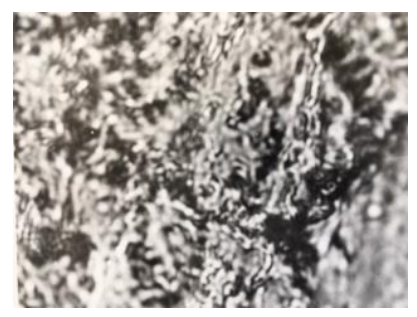

PA

Fig.1. Polarizing optical microscope images of combined PUI/PA dispersions

\section{Conclusions}

Aggregatively stable film-forming polyurethane-polyacrylate aqueous dispersions were obtained by mechanical combinig of different amounts of initial polyurethane and polyacrylate dispersions. It was found that the obtained colloidal mixtures retain the level of dispersion characteristic of individual components.

According to the data of rheological studies, polyurethane-polyacrylate mixtures are structured non-Newtonian systems in the entire studied range of compositions and are characterized by thixotropic properties.

The strength of binary films exceeds that of individual components, wherein the range of increased strength values coincides with the range of thermodynamic compatibility of the components.

Thus, the properties of the combined mixtures can be controlled by changing their quantitative composition. 